\title{
First report of leaf spots caused by Pestalotiopsis trachicarpicola in Gentiana rhodantha
}

\section{Xiaoyong Zhang ( $\nabla$ swuxiaoyong@sina.com )}

Liupanshui Normal University https://orcid.org/0000-0002-3642-6068

\section{Youlian Yang}

Liupanshui Normal University

\section{Shujiang Li}

Liupanshui Normal University

Kai Yan

Liupanshui Normal University

Xinran Li

Liupanshui Normal University

\section{Research Article}

Keywords: Leaf spot, Gentiana rhodantha, Pestalotiopsis trachicarpicola, Taxonomy

Posted Date: April 16th, 2021

DOI: https://doi.org/10.21203/rs.3.rs-320886/v1

License: @) (i) This work is licensed under a Creative Commons Attribution 4.0 International License. Read Full License 


\section{Abstract}

In August 2018, leaf spots were widely found with circular or irregular brown lesions on Gentiana rhodantha leaves in Liupanshui, Guizhou Province, China. Three strains of Pestalotiopsis spp were associated with leaf lesions by single-spore isolation. Based on asexual and sexual characteristics and multi-locus (ITS, TUB, tef-1) phylogenies data, strains were identified as Pestalotiopsis trachicarpicola. This is the first report of G. rhodantha leaf spot caused by P. trachicarpicola. The fungal culture tests showed that mycelia optimum growth temperature and $\mathrm{pH}$ were $20-25^{\circ} \mathrm{C}$ and $\mathrm{pH} 7-9$, and for conidia germination were $25-30^{\circ} \mathrm{C}$ and $\mathrm{pH} 6-9$, respectively.

\section{Main Text}

Gentian rhodantha Franch. ex Hemsl, a perennial herb belonging to the Gentianaceae, is mainly distributed at high altitudes (900-1800 m) in mountainous areas in southwest China. The aerial parts of this herb (including flower, leaf, and stem) are widely used in Tibetan and Miao traditional ethnomedicine for the treatment of cough, bronchitis, hepatitis, and dysentery (Xu et al. 2011).

In August 2018, leaf spots were widely observed in a survey of cultivated field of $G$. rhodantha in Liupanshui, Guizhou Province, China. The typical symptoms on leaves were small brown spots in center or edge of the leaves, which expand to circular or irregular spots with purple halo (Fig. 2a) and even completely wither. In this study, three Pestalotiopsis-like strains (LBB062904, LBB062905 and LBB062906) were isolated from leaf spots of $G$. rhodantha field by single-spore isolation (Chomnunti et al. 2011).

For molecular analysis, total genomic DNA of fresh pure cultures were extracted using the SDS-CTAB method (Suwannarach et al. 2010). The internal transcribed spacers (ITS) region of rDNA molecule was amplified using primer pairs ITS5 and ITS4, the $\beta$-tubulin gene (TUB) region with BT2A and BT2B primer pairs, and the translation elongation factor 1-alpha (tef1) gene using the EF1-728F/EF2 (Maharachchikumbura et al. 2012). PCR was performed with the $25 \mu l$ reaction system consisting of 19.75 $\mu \mathrm{l}$ of double distilled water, $2.5 \mu \mathrm{l}$ of $10 \times$ Taq buffer with $\mathrm{MgCl}_{2}, 0.5 \mu \mathrm{l}$ of dNTP (10 mM each), $0.5 \mu \mathrm{l}$ of each primer $(10 \mu \mathrm{M}), 0.25 \mu \mathrm{l}$ Taq DNA polymerase $(5 \mathrm{U} / \mu \mathrm{l})$, and $1.0 \mu \mathrm{l}$ of DNA template. PCR amplification protocols were performed as described by Maharachchikumbura et al. (2012). The DNA sequences obtained in this study have been deposited in the DDBJ/EMBL/GenBank database with accession numbers MT539135 to MT539137 and MT671942 to MT671947.

Phylogenetic tree was constructed by MEGA X (Kumar et al. 2018) for the combined data set of the ITS, tef1 and TUB genes for 31 Pestalotiopsis strains (Table 1), P. camelliae CBS 443.62 was used as outgroup. Bootstrap value $\geq 50 \%$ (1000 replications) by the maximum likelihood (ML) methods were shown on the respective branch. The aligned results comprised 1413 characters including partial gaps (ITS: 1509, tef1: 510-985 and TUB: 986-1413). Of these characters, 1275 were constant, 54 were variable and 106 were parsimony-informative. 31 Pestalotiopsis strains formed a strong clade (100\% bootstrap support). Three strains obtained were clustered to $P$. trachicarpicola with an $82 \%$ bootstrap value (Fig. 1).

Strains sporulation were observed on synthetic nutrient-poor agar (SNA) amended with double-autoclaved pine needles placed on the agar surface, and incubated at $25^{\circ} \mathrm{C}$ for 15 days (Liu et al. 2017). Doubleautoclaved Taxus chinensis needle was used to induce sexual forms, and the strains were inoculated in the center of SNA plates that contained needles and incubated at $20^{\circ} \mathrm{C}$ for 8 weeks.

The colonies on potato dextrose agar (PDA) were $7.30 \pm 0.51 \mathrm{~cm}(\mathrm{n}=5)$ diameter at $25^{\circ} \mathrm{C}$ after 5 days, with undulate and redial edge, convex with plat surface, white to faint yellow on front, back pale honey-colored (Fig. 2b, c). Conidiomata pycnidial at pine needles in culture on SNA, globose, scattered, black, teardropform, 100-500 $\mathrm{mm}$ diameter (Fig. 2d-e); Conidiophores short, subcylindrical, hyaline, smooth, (Fig. 2f). Conidiogenous cells ampulliform, hyaline, 7.3-14.5×2.3-3.8 $\mu \mathrm{m}(\square \mathrm{x} \pm \mathrm{SD}=8.23 \pm 4.33 \times 2.56 \pm 0.78 \mu \mathrm{m}$, $\mathrm{n}=15)$. Conidia fusoid, olivaceous to thin yellow, 4-septate, $20-26 \times 5-9 \mu \mathrm{m}(\square \mathrm{x} \pm \mathrm{SD}=22.9 \pm 1.3 \times 6.5 \pm 0.8$ 
$\mu \mathrm{m}, \mathrm{n}=30$ ), thin-walled and verruculose wall (Fig. 2g-j); Basal cell obconic, thin-walled, hyaline, 3.5-6.4 $\mu \mathrm{m}$ long; Three median cells dolioform, versicolor, 13-17 $\mu \mathrm{m}$ long $(\square \mathrm{x} \pm \mathrm{SD}=15.1 \pm 0.7 \mu \mathrm{m}, \mathrm{n}=30$ ), verruculose, pale-yellow to brown, septa darker; apical cell conical, hyaline, smooth wall, 2.8-5.5 $\mu \mathrm{m}$ long, with 1-4 concurrent tubular apical appendages (mostly 3), filiform, unbranched, arising from the apical crest, 8-21 $\mu \mathrm{m}$ long; single basal appendage is straight, 3-7 $\mu \mathrm{m}$ long.

Ascomatas on Taxus chinensis needle were 130-320 $\mathrm{\mu m}$ diam, black, gregarious, immersed in leaves epidermis and raised slightly (Fig. 2k). Asci 8-spored, bitunicate, cylindrical, 67-94×6-10 $\mu \mathrm{m}$ $(\square \mathrm{x} \pm \mathrm{SD}=84.8 \pm 7.6 \times 9.4 \pm 1.3 \mu \mathrm{m}, \mathrm{n}=30)$ (Fig. $2 \mathrm{l}-\mathrm{m})$, mature ascus without wall, indistinct J+ (amyloid ring) in apical apparatus (Fig. 2n). Ascospores 13.5-15.2×5.1-7.5 $\mu \mathrm{m}(\square \mathrm{x} \pm \mathrm{SD}=14.3 \pm 0.9 \times 6.5 \pm 0.4 \mu \mathrm{m}, \mathrm{n}=30)$, uniseriate or interlaced, 2-4 cells (mostly 3) ascospore concolorous, light yellow to brown, oblong to fusiform, smooth or verrucose, brown septate and slightly constricted at the septa(Fig. 2o-r). Based on molecular analysis and unsexual and sexual characters, three strains were identified as $P$. trachicarpicola Y.M. Zhang \& K.D. Hyde (Zhang et al. 2012), To the best of my knowledge, this is the first report of $P$. trachicarpicola causing leaf spot of $G$. rhodantha.

$P$. trachicarpicola have many asexual features similar to P. neglecta, $P$. kenyana and $P$. oryzae, which have thin and olivaceous walled conidia, concolourous medial cells and branches of apical appendages. But there are some significant differences between them (Table 2). The conidia of $P$. trachicarpicola $(\square \mathrm{x}=22.9 \times 6.5$ $\mu \mathrm{m})$ are shorter than those of $P$. kenyana $(\square \mathrm{x}=25.5 \mu \mathrm{m})$ and $P$. oryzae $(\square \mathrm{x}=26.9 \mu \mathrm{m})$ and the apical appendages of $P$. trachicarpicola $(8-21 \mu \mathrm{m})$ are shorter than $P$. neglecta and $P$. oryzae (Maharachchikumbura et al. 2014; Steyaert, 1953; Zhang et al. 2012). The asci and ascospores of $P$. trachycarpicola is morphologically mostly similar to $P$. neglecta and $P$. accidenta, but the asci of $P$. trachycarpicola are thinner than $P$. neglecta, and ends of the ascospores of $P$. trachicarpicola are more rounded and some are verrucose compare with $P$. neglecta and $P$. accidenta (Kobayashi et al.2001; Zhu et al, 1991).

In pathogenicity tests, detached leaves of G. rhodantha were surface-disinfected with $75 \%$ EtOH for $30 \mathrm{~s}$ and washed five times with sterilized water. Leaves were slightly wounded with a sterile needle and then inoculated a $2 \mathrm{~mm}$ diam PDA mycelial disk and inoculated sterilized PDA as controls and placed in Petri dishes at $25^{\circ} \mathrm{C}$. There were 10 leaves for each treatment and performed three times. The inoculated leaves had observed symptoms consisted of circular, brown to black spots and highly similar to pathology characteristics of the specimen at 7th days after inoculation (Fig. 2s-t). None of the control leaves had symptoms. The same fungus was re-isolated from symptomatic lesion.

To determine the optimal temperature and $\mathrm{pH}$ on spore germination and mycelial growth of $P$. trachicarpicola, eight temperature gradients $\left(5-40^{\circ} \mathrm{C}\right)$ and ten $\mathrm{pH}$ values $(3.0-12.0)$ of $\mathrm{PDA}$ medium were designed as a randomized single factor experiment. All pates for $\mathrm{pH}$ tests were cultured at $25^{\circ} \mathrm{C}$. Experiments were conducted 3 times in the dark. Germination rate were calculated we counted the number of total and germinated spore in 10 visions under low-power microscopic $(10 \times 10)$ at $12 \mathrm{~h}$, and measured the diameters of colony after 7 days. The results showed that the mycelia grew at a temperature range of 10 to $35^{\circ} \mathrm{C}$, with optimum growth at 20 to $25^{\circ} \mathrm{C}$. The conidia sprout had a temperature range of $15-40^{\circ} \mathrm{C}$, and the optimum temperature were $25-30^{\circ} \mathrm{C}$ (Fig. 3a). After treatment of medium with different $\mathrm{pH}$ values, mycelia could grow at pH 4 to 12, with optimum growth rate and conidia germination rate at 8 and 6-9, respectively (Fig. 3b).

Brown leaf spot and leaf blight caused by Mycochaetophora gentianae (Nekoduka et al. 2013) and Septoria gentianae (Verkley et al. 2013) were two serious disease on overground parts of Gentiana plants and widely occurred in fields, but they symptoms were significantly different with leaf spot in size, color and edge type in focuses. There were without any records of Pestalotiopsis diseases on wild and cultivated G. rhodantha yet. Therefore, we propose that leaf spot caused by $P$. trachicarpicola be added as novel disease on G. rhodantha.

\section{Declarations}


Acknowledgements The research was funded by Youth Science and Technology Talent Cultivating Project of Guizhou Department of Education (no. QJH KY[2018]381), Construction Project of Key and Distinctive Laboratory of Guizhou General University (no. QJH KY2017[012]) and Natural Science Foundation of Guizhou Province (no. QKH J[2014]7447). We thank EditSprings (https://www.editsprings.com/) for providing expert linguistic services.

\section{Compliance with ethical standards}

Conflict of interest The authors declare that they have no conflict of interest.

Ethical approval This article does not contain any studies with human participants or animals performed by any of the authors.

\section{References}

Chomnunti P, Schoch CL, Aguirre-Hudson B, Ko-Ko TW, Hongsanan S, Jones EBG, Kodsueb R, Phookamsak R, Chukeatirote E, Bahkali AH, Hyde KD (2011) Capnodiaceae. Fungal Divers 51:103-134

Kobayashi T, Ishihara M, Ono Y (2001) A new species of Pestalosphaeria, the teleomorph of Pestalotiopsis neglecta. Mycoscience 42:211-216

Kumar S, Stecher G, Li M, Knyaz C, Tamura K (2018) MEGA X: Molecular evolutionary genetics analysis across computing platforms. Mol Biol Evol 35:1547-1549

Liu F, Hou L, Raza M, Cai L (2017) Pestalotiopsis and allied genera from camellia, with description of 11 new species from China. Sci Rep 7:1-19

Maharachchikumbura SSN, Guo LD, Cai L, Chukeatirote E, Wu WP, Sun X, Crous PW, Bhat DJ, McKenzie EHC, Bahkali AH, Hyde KD (2012) A multi-locus backbone tree for pestalotiopsis, with a polyphasic characterization of 14 new species. Fungal Divers $56: 95-129$

Maharachchikumbura SSN, Hyde KD, Groenewald JZ, Xu J, Crous PW (2014) Pestalotiopsis revisited. Stud Mycol 79:121-186

Nekoduka S, Tanaka K, Sano T (2013) Overwintering of brown leaf spot fungus, Mycochaetophora gentianae, in infected gentian leaves as the primary inoculum source. J Gen Plant Pathol 79:175-177

Steyaert RL (1953) New and old species of Pestalotiopsis. Transactions of the British Mycological Society 36:81-89

Suwannarach N, Bussaban B, Hyde KD, Lumyong S (2010) Muscodor cinnamomi, a new endophytic species from Cinnamomum bejolghota. Mycotaxon 114:15-23

Verkley GJ, Quaedvlieg W, Shin HD, Crous PW (2013) A new approach to species delimitation in Septoria. Stud Mycol 75:213305

Xu M, Zhang M, Wang D, Yang CR, Zhang YJ (2011) Phenolic compounds from the whole plants of Gentiana rhodantha (Gentianaceae). Chem Biodivers 8:1891-1900

Zhang YM, Maharachchikumbura S, Mckenzie E, Hyde KD (2012) A novel species of Pestalotiopsis causing leaf spots of Trachycarpus fortune. Cryptogamie Mycol 33:311-318

Zhu P, Ge Q, Xu T (1991) The perfect stage of Pestalotiopsis from China. Mycotaxon 40:129-140

\section{Tables}


Table 1 Strains used in this study with host, location, and GenBank accession number

\begin{tabular}{|c|c|c|c|c|c|c|}
\hline \multirow[t]{2}{*}{ Species } & \multirow[t]{2}{*}{ Strain/isolation No. } & \multirow[t]{2}{*}{ Host/Substrate } & \multirow[t]{2}{*}{ Location } & \multicolumn{3}{|c|}{ GenBank accession } \\
\hline & & & & ITS & tef1 & TUB \\
\hline \multirow[t]{2}{*}{ P. neglecta } & TAP99M112 & Pieris japonica & Japan & $\mathrm{AB} 482211$ & $\mathrm{AB} 453853$ & AB453882 \\
\hline & TAP200063 & Cupressus macrocarpa & Japan & AB482209 & AB453889 & AB453841 \\
\hline \multirow[t]{3}{*}{ P. oryzae } & CBS 171.26 & Unknown & Italy & MH854881 & KM199494 & KM199397 \\
\hline & CBS 353.69 & Oryza & Denmark & KM199299 & KM199496 & KM199398 \\
\hline & CBS $111522^{*}$ & Telopea & Hawaii & KM199294 & KM199493 & KM199394 \\
\hline \multirow[t]{6}{*}{ P. trachicarpicola } & LBB062904 & Gentiana rhodantha & China & MT539135 & MT671942 & MT671945 \\
\hline & LBB062905 & Gentiana rhodantha & China & MT539136 & MT671943 & MT671946 \\
\hline & LBB062906 & Gentiana rhodantha & China & MT539137 & MT671944 & MT671947 \\
\hline & OP068* & Trachycarpus fortunei & China & JQ845947 & JQ845946 & JQ845945 \\
\hline & CBS 111507 & Unknown & Zimbabwe & MH553960 & MH554378 & MH554619 \\
\hline & CBS 297.76 & Soil & Spain & MH554027 & MH554462 & MH554704 \\
\hline \multirow[t]{3}{*}{ P. kenyana } & CBS $442.67^{*}$ & Coffea & Kenya & MH859026 & MH870724 & KM199395 \\
\hline & CBS 911.96 & Agar & Unknown & KM199303 & KM199503 & KM199396 \\
\hline & LC6633 & Camellia sinensis & China & KX895027 & KX895246 & KX895360 \\
\hline \multirow[t]{2}{*}{ P. rhodomyrtus } & LC4458* & Camellia sinensis & China & KX895010 & KX895228 & KX895342 \\
\hline & LC3413 & Camellia sinensis & China & KX894981 & KX895198 & KX895313 \\
\hline \multirow[t]{2}{*}{ P. australasiae } & CBS $114126^{*}$ & Knightia sp. & New Zealand & KM199297 & KM199499 & KM199409 \\
\hline & CBS 114141 & Protea sp. & Australia, & KM199298 & KM199501 & KM199410 \\
\hline \multirow[t]{2}{*}{ P. telopeae } & CBS 114137 & Protea & Australia & KM199301 & KM199559 & KM199469 \\
\hline & CBS $113606^{*}$ & Telope & Australia & KM199295 & KM199498 & KM199402 \\
\hline \multirow[t]{2}{*}{ P. brachiata } & LC2988 & Camellia sp. & China & KX894933 & KX895150 & KX895265 \\
\hline & LC8189* & Camellia sp. & China & KY464143 & KY464153 & KY464163 \\
\hline \multirow[t]{2}{*}{ P. biciliata } & CBS 790.68 & Unknown & Netherlands & MH859228 & KM199507 & KM199400 \\
\hline & CBS $124463^{*}$ & Platanusx hispanica & Slovakia & KM199308 & KM199505 & KM199399 \\
\hline \multirow[t]{2}{*}{ P. disseminata } & CBS 143904 & Persea americana & New Zealand & MH554152 & MH554587 & MH554825 \\
\hline & CBS $118552^{*}$ & Eucalyptus botryoides & New Zealand & MH553986 & MH554410 & MH554652 \\
\hline \multirow[t]{2}{*}{ P. adusta } & MFLUCC10-0146* & Prunus cerasus & USA & X399007 & JX399071 & JX399038 \\
\hline & ICMP6088 & Unknown & Fiji & JX399006 & JX399070 & JX399037 \\
\hline \multirow[t]{2}{*}{ P. knightiae } & CBS 111963 & Knightia sp. & New Zealand & KM199311 & KM199495 & KM199406 \\
\hline & CBS $114138^{*}$ & Knightia sp. & New Zealand & KM199310 & KM199497 & KM199408 \\
\hline P. camelliae & CBS $443.62^{*}$ & camelliae & China & MH858206 & KM199512 & KM199424 \\
\hline
\end{tabular}

* means type strain, epitype strain or outgroup strain.

Table 2 Sexual and asexual forms of P. trachicarpicola and its related species

\begin{tabular}{|c|c|c|c|c|c|c|c|c|c|c|}
\hline \multirow[t]{3}{*}{ Species/strain } & \multicolumn{6}{|c|}{ Anamorph } & \multicolumn{4}{|c|}{ Teleomorph } \\
\hline & \multirow{2}{*}{$\begin{array}{c}\text { Conidia } \\
\text { size }(\mu \mathrm{m})\end{array}$} & \multirow{2}{*}{$\begin{array}{c}\text { Color of three } \\
\text { medial cell }\end{array}$} & \multicolumn{2}{|c|}{ Apical appendage } & \multicolumn{2}{|c|}{ Basal appendage } & \multirow{2}{*}{$\begin{array}{c}\text { Asci } \\
\text { size }(\mu \mathrm{m})\end{array}$} & \multicolumn{3}{|c|}{ Ascospore } \\
\hline & & & $\begin{array}{l}\text { Length } \\
(\mu \mathrm{m})\end{array}$ & Quantity & $\begin{array}{l}\text { Length } \\
(\mu \mathrm{m})\end{array}$ & Quantity & & Size $(\mu \mathrm{m})$ & $\begin{array}{c}\text { No. of } \\
\text { Septate }\end{array}$ & Wall \\
\hline $\begin{array}{l}P . \\
\text { trachicarpicola } \\
\text { LBB062904 }\end{array}$ & $\begin{array}{l}20-26 \times 5- \\
9 \\
(22.9 \times 6.5) \\
a\end{array}$ & $\begin{array}{l}\text { Concolourous, } \\
\text { olivaceous to } \\
\text { light yellow }\end{array}$ & $8-21$ & $\begin{array}{l}1-4 \\
\text { (mostly } \\
3 \text { ) }\end{array}$ & $3-7$ & 1 & $\begin{array}{l}67-94 \times 6- \\
10 \\
(84.8 \times 9.4)\end{array}$ & $\begin{array}{l}13.5- \\
15 \times 5-7.5 \\
(14.3 \times 6.5)\end{array}$ & $\begin{array}{l}1-3 \\
\text { (mostly } \\
2 \text { ) }\end{array}$ & $\begin{array}{l}\text { Smooth } \\
\text { or } \\
\text { verrucose }\end{array}$ \\
\hline $\begin{array}{l}P . \\
\text { trachicarpicola } \\
\text { OP068 b }\end{array}$ & $\begin{array}{l}19- \\
24.9 \times 5- \\
6.3 \\
(22 \times 6.0)\end{array}$ & $\begin{array}{l}\text { Concolorous, } \\
\text { olivaceous }\end{array}$ & $\begin{array}{l}9.4- \\
17.8\end{array}$ & $\begin{array}{l}2-3 \\
\text { (mostly } \\
3 \text { ) }\end{array}$ & $2.8-11$ & $\begin{array}{l}1-2 \\
\text { (mostly } \\
1 \text { ) }\end{array}$ & $\begin{array}{l}65-76 \times 5- \\
14 \\
(73.6 \quad \times \\
9.3)\end{array}$ & $\begin{array}{l}12-16 \times 5- \\
8 \\
(14.1 \times 6.5)\end{array}$ & $\begin{array}{l}2-3 \\
\text { (mostly } \\
2 \text { ) }\end{array}$ & $\begin{array}{l}\text { Smooth } \\
\text { or } \\
\text { verrucose }\end{array}$ \\
\hline P. kenyana ${ }^{\mathrm{c}}$ & $\begin{array}{l}23-28 \times 7- \\
9 \\
(25.5 \times 8)\end{array}$ & $\begin{array}{l}\text { Concolourous, } \\
\text { olivaceous }\end{array}$ & $3-20$ & $2-3$ & $1-4$ & $\begin{array}{l}1-2 \\
\text { (mostly } \\
2 \text { ) }\end{array}$ & - & - & - & - \\
\hline $\begin{array}{l}\text { P. neglecta }{ }^{\mathrm{d}} \\
\left(\text { Ps. gubae }{ }^{\mathrm{e}}\right)\end{array}$ & $\begin{array}{l}19-25 \times 6- \\
8 \\
(21 \times 6.6)\end{array}$ & $\begin{array}{l}\text { Concolourous, } \\
\text { brown }\end{array}$ & $9-22$ & $\begin{array}{l}2-3 \\
\text { (mostly } \\
3 \text { ) }\end{array}$ & $0-7$ & 1 & $\begin{array}{l}75- \\
88 \times 10-12\end{array}$ & $\begin{array}{l}9.5- \\
17.5 \times 5-7\end{array}$ & $\begin{array}{l}2-3 \\
\text { (mostly } \\
2 \text { ) }\end{array}$ & Smooth \\
\hline P. oryzae ${ }^{\mathrm{C}}$ & $\begin{array}{l}24.5-29 \times \\
6-8 \\
(26.9 \times 7)\end{array}$ & $\begin{array}{l}\text { Concolourous, } \\
\text { olivaceous }\end{array}$ & $9-27$ & $\begin{array}{l}2-3 \\
\text { (mostly } \\
3 \text { ) }\end{array}$ & $3-6$ & 1 & - & - & - & - \\
\hline $\begin{array}{l}\text { P. baarnensis } \\
(\text { Ps. } \\
\text { accidenta })^{\mathrm{f}}\end{array}$ & $\begin{array}{l}16.5- \\
27 \times 5.6- \\
6.7\end{array}$ & $\begin{array}{l}\text { Concolourous, } \\
\text { reddish brown }\end{array}$ & $\begin{array}{l}10- \\
32.5\end{array}$ & $\begin{array}{l}2-3 \\
\text { (mostly } \\
2 \text { ) }\end{array}$ & $\begin{array}{l}2.3- \\
6.8\end{array}$ & 1 & $\begin{array}{l}62.5- \\
72.9 \times \\
8.3-8.9\end{array}$ & $\begin{array}{l}13- \\
17 \times 4.8- \\
6.8\end{array}$ & $\begin{array}{l}1-3 \\
\text { (mostly } \\
2 \text { ) }\end{array}$ & Smooth \\
\hline
\end{tabular}

a The average values of length and width were listed in brackets.

b Zhang et al. 2012

C Maharachchikumbura et al. 2014

d Steyaert, 1953

e Kobayashi et al.2001

f Zhu et al. 1991 


\section{Figures}

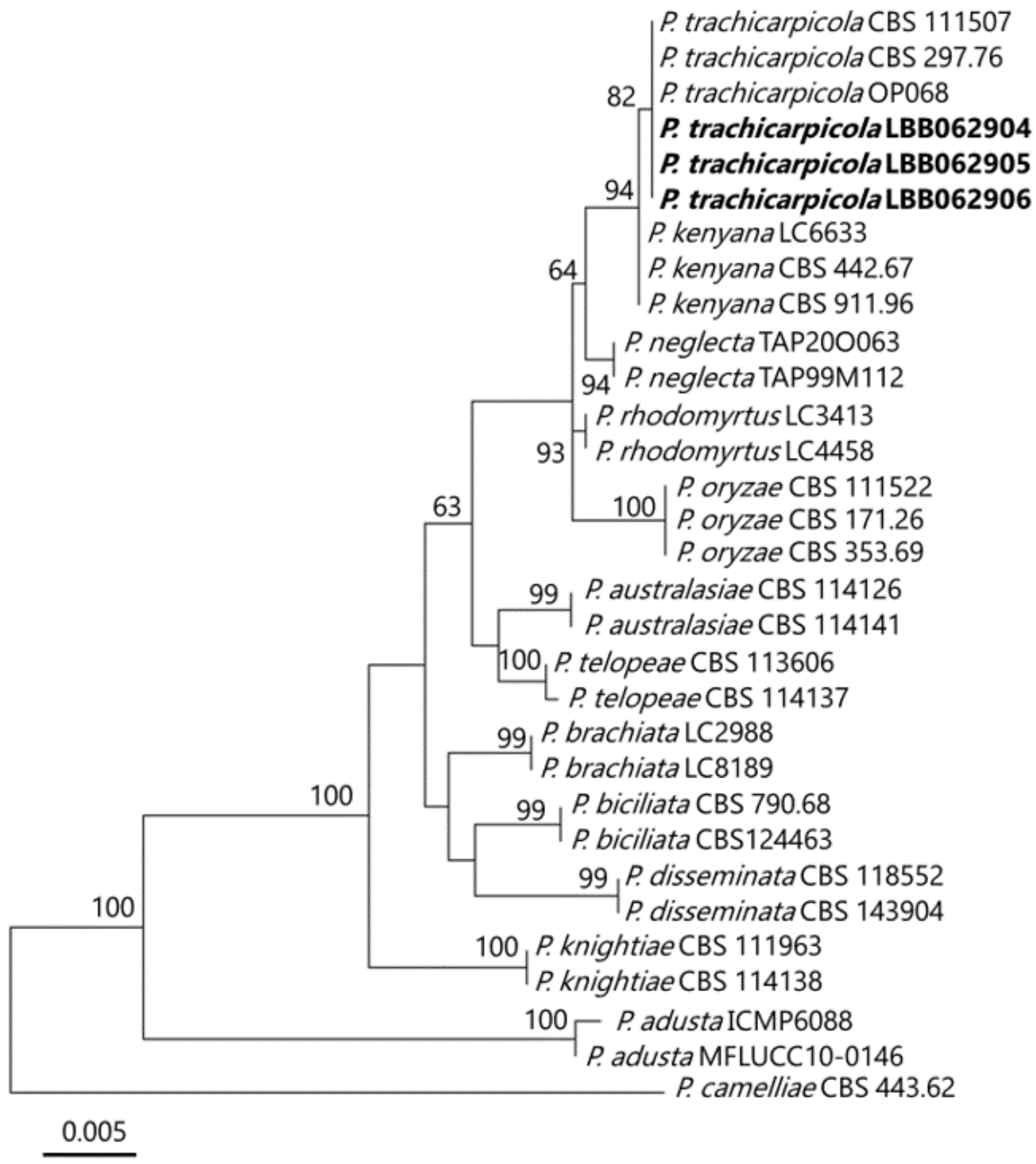

Figure 1

Topology showing the maximum likelihood tree, inferred from combined ITS, TUB and tef1 gene regions. Bootstrap values smaller than $50 \%$ are not shown. Strain Pestalotiopsis camelliae CBS 443.62 was used as the outgroup taxon. 3 strains from G. trachicarpicola in this study are thickened. 


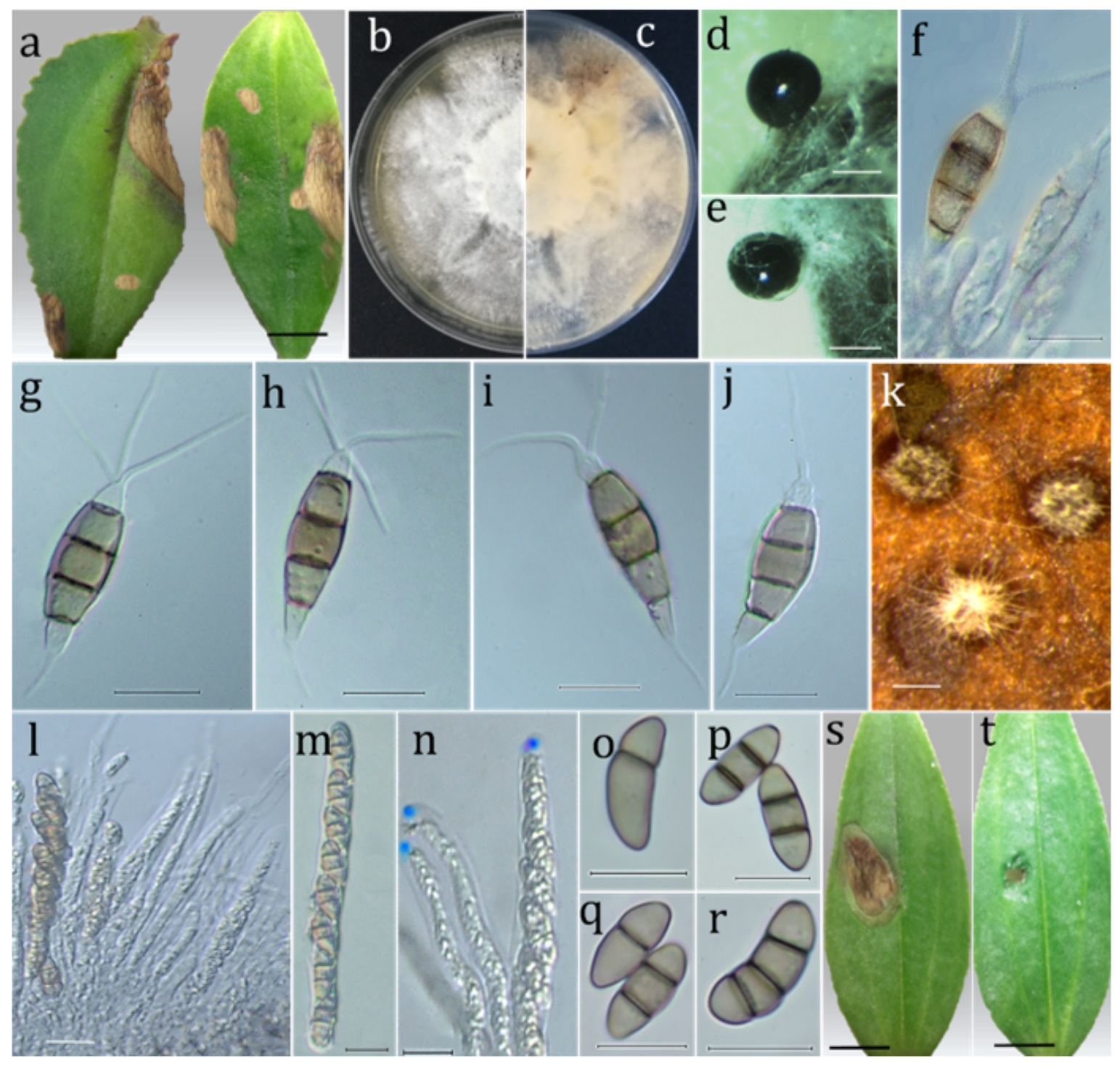

Figure 2

Symptoms and pathogen morphology of leaf spot in Gentiana rhodantha caused by Pestalotiopsis trachicarpicola. a Symptoms of leaf spots on leaf of G. rhodantha, scale bar=5.0 mm. b-c Front and back view of colony on PDA. d-e Acervuli on double-autoclaved pine needle, scale bars $=200 \mu \mathrm{m}$. $\mathrm{f}$ Conidiophore, conidiogenous cells and developing conidia. $\mathrm{g}-\mathrm{j}$ Conidia, scale bars $=10 \mu \mathrm{m}$. $\mathrm{k}$ Ascomatas on double-autoclaved Taxus chinensis needle with SNA medium, scale bar=200 $\mu \mathrm{m}$. I-n Mature and immature unitunicate asci and amyloid ring on apical apparatus, scale bars $=10 \mu \mathrm{m}$. $0-r$ Ascospores, scale bars $=10 \mu \mathrm{m}$. s Symptoms after inoculation with Pestalotiopsis trachicarpicola, and t Control at 7th days, scale bar=5.0 mm. 


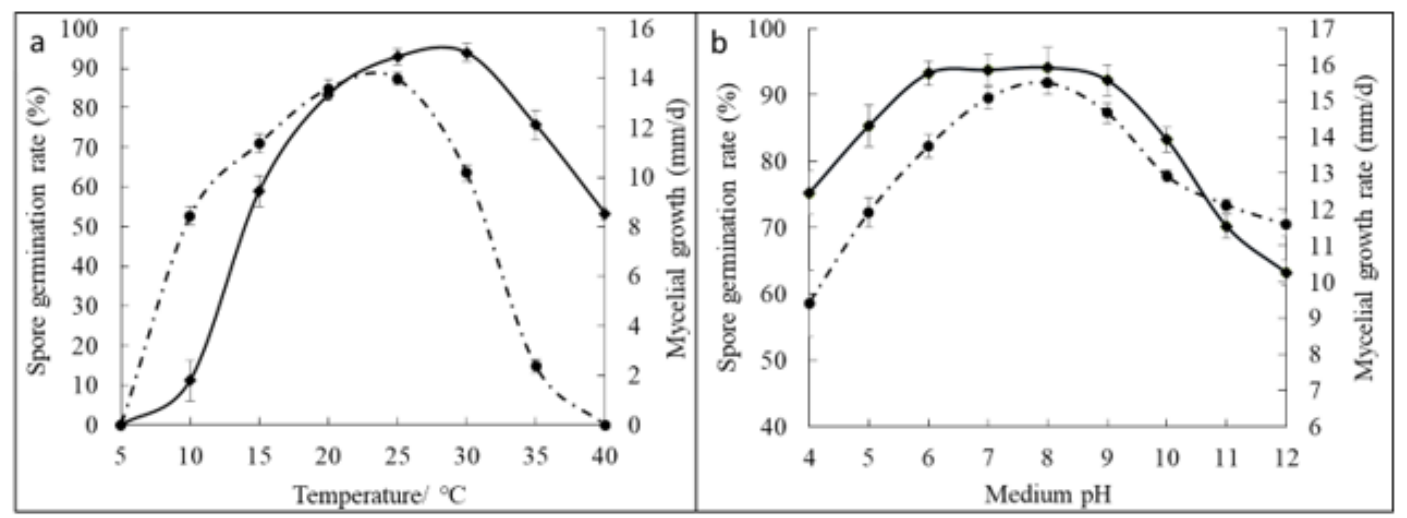

$\rightarrow-$ Spore germination rate $-\bullet-$ Mycelial growth rate

\section{Figure 3}

Effect of temperature and $\mathrm{pH}$ value on Pestalotiopsis trachicarpicola mycelial growth and spore germination 